\title{
ÊTRE EMPLOYEUR BÉNÉVOLE DANS UNE CRÈCHE ASSOCIATIVE ET PARENTALE
}

\author{
Simon Cottin-Marx
}

Champ social | « Le Sociographe »

2021/1 N 73 | pages 53 à 65

ISSN 1297-6628

DOI 10.3917/graph.073.0053

Article disponible en ligne à l'adresse :

https://www.cairn.info/revue-le-sociographe-2021-1-page-53.htm

Distribution électronique Cairn.info pour Champ social.

(C) Champ social. Tous droits réservés pour tous pays.

La reproduction ou représentation de cet article, notamment par photocopie, n'est autorisée que dans les limites des conditions générales d'utilisation du site ou, le cas échéant, des conditions générales de la licence souscrite par votre établissement. Toute autre reproduction ou représentation, en tout ou partie, sous quelque forme et de quelque manière que ce soit, est interdite sauf accord préalable et écrit de l'éditeur, en dehors des cas prévus par la législation en vigueur en France. Il est précisé que son stockage dans une base de données est également interdit. 


\section{Être employeur bénévole dans une crèche associative et parentale}

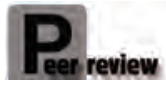

Simon Cottin-Marx

L

a sociologie s'est longtemps intéressée au bénévolat comme un engagement. Depuis quelques années, les sociologues du travail associatif sont venus renouveler cette approche et proposent de regarder les bénévoles comme des travailleurs (Simonet, 2004; 2010; Hély et Simonet, 2013). Dans la continuité de ces travaux, nous proposons d'étudier les bénévoles, qui siègent dans les instances de direction des associations employeuses, non seulement comme des travailleurs, mais aussi comme des employeurs. Car c'est une des spécificités du monde associatif employeur : les " patron.nes " (Offerlé, 2017) des salarié.es sont les président.es, et plus largement les membres (forcément) bénévoles qui siègent dans les conseils d'administration. Pour nommer les structures qu'ils dirigent, les sociologues du travail associatif ont forgé la notion "d'entreprise associative " (Marchal, 1992 ; Hély, 2009). Celle-ci permet de souligner que ces structures juxtaposent deux dispositifs distincts. Avec d'une part, celui de l'association, fondée sur la gestion désintéressée et le travail bénévole. Et d'autre part, celui de l'entreprise employeuse et les obligations attachées au Code du travail. Les dirigeant.es bénévoles de ces entreprises associatives ont donc une position ambivalente. Ils sont " patrons " sans être propriétaires de la structure, et ils sont bénévoles, mais avec la charge de la fonction employeur (Cottin-Marx, 2020). Si celle-ci est souvent déléguée à des directeurs ou des directrices dans les grandes associations, c'est plus rarement le cas dans les petites et très petites associations employeuses ( $<10$ salarié.es) (Cottin-Marx, 2016). Ce sont alors les responsables bénévoles de la structure qui la mettent en œuvre, parfois de manière partagée avec des salarié.es ou des prestataires (le comptable par exemple). C'est eux qui, collectivement, 
embauchent, signent les contrats, éditent les feuilles de paie, versent les salaires, sont responsables de l'organisation et des conditions de travail, etc.

Le rôle et les responsabilités de ces employeurs bénévoles ont été peu explorés, même si l'on peut remarquer les travaux notables des sociologues Matthieu Hély, Maud Simonet, Sophie Rétif (2015) ou encore des économistes Emmanuelle Puissant et Anne Le Roy (2019). Dans cet article, nous proposons d'approfondir ce sujet en nous intéressant aux bénévoles qui occupent la fonction employeur au sein d'une crèche parentale. Qu'est-ce qui amène des bénévoles à devenir " patrons »? En quoi consiste la fonction employeur? De quelle manière l'occupent-ils? C'est quoi être employeur bénévole? Pour répondre à ces questions, nous nous appuierons sur notre propre expérience dans cette structure, sur cinq entretiens exploratoires et surtout sur l'exploitation de cinq entretiens semi-directifs réalisés avec des responsables bénévoles (sur la période 2016-2019) de cette organisation régie par la loi 1901.

\section{4 - Présentation de la crèche parentale «Badaboum »}

La crèche parentale "Badaboum " (1) est située en Île-de-France. Conventionnée par la Caisse des allocations familiales (CAF), elle est membre de l'ACEPRIF (2) et accueille 19 enfants, ayant entre 6 mois et 3 ans. Cette structure a pour particularité d'être associative et parentale, c'est-à-dire que les parents sont des usagers de l'association, ils en sont aussi adhérents, participent à son activité et à sa gestion économique et sociale.

Concrètement, les 19 familles réalisent chaque semaine une demijournée ( 5 heures) de "présence » auprès des enfants (pas forcément auprès de leur enfant), où ils accomplissent diverses tâches (ménage, activités pour enfants, surveillance, etc.) en soutien à l'équipe professionnelle (composée de deux EJE [Éducateurs de jeunes enfants], d'une auxiliaire de puériculture, de trois assistantes petites enfants,

(1) Pour respecter la confidentialité des entretiens, le nom de la structure et des personnes ont été anonymisés.

(2) Association des collectifs enfants-parents-professionnel.les de la Région Île-deFrance. 
d'une cuisinière et d'un agent d'entretien et de service). Au total, le temps de "présence " parental représente ainsi $90 \mathrm{~h}$ par semaine, soit plus de 2,5 postes Équivalents temps plein (ETP).

Les parents s'occupent également collectivement de la gestion de la crèche, dans le cadre de réunions plénières ( 2 à 5 heures par mois par famille) et de " commissions" aux missions bien définies. Un travail non négligeable qu'ils évaluent à $5 \mathrm{~h}$ par semaine et par famille. Les membres de la commission " parents " réalisent par exemple les plannings des présences et s'occupent des commandes de "renforts " (de parents lorsqu' une professionnelle est absente - ce qui arrive régulièrement). La commission " course " s'occupe de l'approvisionnement en nourriture et en matériel de la crèche, etc. Au total, les parents sont organisés au sein de 8 commissions (3), mais ce sont deux "grosses commissions", que les parents décrivent comme "chronophages» et entrainant une forte "charge mentale " qui retiennent notre intérêt ici, car c'est là que se concentre la "fonction employeur". Il s'agit en premier lieu du "bureau ". Ses 6 membres s'occupent de la gestion quotidienne de la crèche avec la Responsable technique (RT) et son adjointe (RTA) (4), des relations parents-équipe, de la coordination du travail des commissions, de l'animation de la vie associative (Assemblées générales et intermédiaires, réunions parents-équipe, etc.), des relations avec les partenaires, etc. La commission "salariés », également composée de 6 parents (majoritairement des mères), gère quant à elles toutes les questions relatives à l'équipe de 8 salariées (dont presque 7 ETP). Cette

(3) Les autres commissions sont les " coms » : communication (qui s'occupe de la gestion des outils numériques; gestion des téléphones et appareils photos), environnement (organisation des week-ends ménages, veille au respect des normes collectives, organisation de formations aux gestes d'urgence pour l'équipe et les parents, organisation des exercices d'évacuation, etc.), intendance (organisation, suivi et/ou réalisation des travaux et réparation au sein de la crèche, etc.), enfants (organisation d'ateliers et d'événements pour les enfants; mise en place et suivi des intervenants extérieurs; organisation des fêtes de fin d'année, etc.).

(4) La RT et la RTA ont la responsabilité technique de la crèche et sont responsables de son bon fonctionnement. $25 \%$ de leur temps de travail est dédié au travail administratif (coordination avec les parents, planning, réunions d'équipe, suivi du projet pédagogique...). Le reste du temps, elles interviennent auprès des enfants pour les accompagner dans leur apprentissage de l'autonomie et de la vie sociale. Garantes des règles de sécurité et d'hygiène, elles sont décisionnaires pour tout ce qui concerne l'encadrement et le déroulement de la journée. 
" com» (" commission») s'occupe de l'embauche, du renouvellement des contrats et des contrats aidés, des éléments relatifs à la formation, le suivi des heures, l'édition des feuilles de paie, des arrêts maladie, des congés, mettre à jour le document unique d'évaluation des risques professionnels, faire passer les entretiens d'évaluation annuels, etc.

$\mathrm{La}$ " fonction employeur » est ainsi éclatée entre ces deux principales commissions, entre 12 parents appartenant à 12 familles différentes. Si l'éclatement interne de ces responsabilités n'est pas propre aux entreprises associatives, puisque cette situation se retrouve dans les entreprises privées où l'on observe des transferts de responsabilités entre une pluralité de fonctions (direction, DRH [Direction des ressources humaines], etc.) et d'individus (Le Roy et al., 2019), la spécificité est qu'ici les individus et le collectif employeur sont usagers et bénévoles (5).

\section{C'est avoir "un rôle important à jouer»}

Si les familles rejoignent la crèche parentale, c'est d'abord pour avoir un moyen d'accueil pour leurs enfants, mais c'est aussi, une adhésion 56- au projet associatif qui promeut une pédagogie attentive aux besoins de l'enfant et donne une place aux parents dans sa mise en ouvre. Ainsi, les parents, quelle que soit leur commission, s'engagent en premier lieu pour avoir un moyen de garde pour leurs enfants et pour mettre en œuvre le projet associatif, non pour devenir employeur. Pourtant, ceux du bureau et de la commission salariés vont exercer cette fonction.

Les parents interrogés, expliquent avoir rejoint les deux commissions ayant la charge de la fonction employeur, par "curiosité » (Samy), pour " aider à ce que les choses fonctionnent mieux " (Kloé), ou encore, car au moment du recrutement à la crèche, ils s'étaient positionnés stratégiquement sur ces "grosses commissions " pour lesquelles ils avaient été informés que les volontaires manquaient. Entrer dans ces commissions est donc parfois le résultat d'un choix stratégique pour intégrer la crèche, dans un territoire où les places en accueil collectif sont rares. Pour Camille, qui a eu deux enfants au sein

(5) Pour définir le bénévolat, nous pouvons retenons trois critères : son caractère volontaire, altruiste (tournée vers autrui) et désintéressé (sans bénéficier d'une rémunération). 
de la crèche Badaboum et qui a passé quatre années à la commission salariés, la raison est encore ailleurs : " Je n’ai pas eu le choix de ma commission, car on n'est pas arrivé à la rentrée de septembre. On a remplacé une famille qui quittait la crèche en novembre. "

Si les bénévoles sont informés très tôt des tâches qu'ils ont à faire, ils ne prennent conscience de leur rôle d'employeur et des responsabilités qui leur incombent qu'à un moment fondateur. Samy, qui a passé deux ans au bureau, dont une année en tant que co-président, explique ainsi : "J'ai mis du temps à comprendre que j'étais employeur. C'est en devenant co-président, la deuxième année, en faisant passer les entretiens d'embauche (RT et RTA) que je l'ai compris. " Pour Camille, membre de la commission salariés, c'est une véritable crise qui lui a fait prendre conscience de son rôle dans la crèche. "Je savais que j'avais la fonction $\mathrm{RH}$, mais dans les faits j'ai réalisé que j'étais employeur quand on a dû mettre fin à la période d'essai d'un salarié, car il parlait mal aux autres salariées et il était assez colérique. [...] On a élaboré une stratégie pour lui dire qu'on ne continuait pas avec lui sans qu'il parte au quart de tour. Mais il a commencé à vouloir se battre. [...] Il a fallu le calmer... il a crié à l'injustice et on a appelé une maman avocate pour lui dire que c'était notre droit. C'était dur, mais ça nous a confortés dans le fait qu'il ne fallait pas qu'il reste, qu'il pouvait être un danger. J'ai réalisé qu'on avait un rôle important à jouer. »

\section{Un rôle chronophage et une importante charge mentale}

Kloé est professeure des écoles, mère de trois enfants dont le dernier est à Badaboum. Pendant, un an elle a été membre du bureau et coprésidente. Elle raconte sur son expérience : " J'étais à la com enfant la première année. Tout le monde voulait aller dans cette com, et moi ça me donnait l'impression d'être au taf. Alors j'ai laissé la place. J'avais envie de me frotter à un truc différent de ce que je fais quotidiennement. [...] Il y avait beaucoup de turn-overs au sein de l'équipe de la crèche, ça m'intéressait de me confronter à ça, d'aider les gens à être bien dans leur boulot... Et j'ai pris cher, j'ai souffert. [...] Tout de suite, on fait une réunion avec com salariés. Et j'hallucine. Le trésorier présente une situation financière catastrophique. On s'est même dit qu'on allait devoir virer des gens. Moi qui trouvais intéressant de faire les entretiens de fin d'année... et là, on me dit qu'il va falloir licencier... Je n'en ai pas dormi. " $\mathrm{Si}$, finalement, une solution 
économique va être trouvée pour éviter les licenciements, la crèche va devoir néanmoins baisser drastiquement ses dépenses. Pour trouver des solutions, mais aussi gérer la crèche au quotidien, Kloé va connaître un rythme de travail bénévole soutenu. "On faisait une réunion tous les 10 jours, et ça finissait rarement avant 23 heures. [...] Une réunion de bureau toutes les trois semaines, et une autre avec la RT pour gérer les questions du quotidien. [...] c'était une grosse charge mentale, beaucoup de travail avec 25 mails par jour qui appelaient des réponses. [...] Je me rappelle que mes collègues étaient tenus en haleine par ce qui se passait à la crèche, ils étaient au courant de l'enjeu des réunions, si j'avais dormi ou pas. C'était passionnant, mais très lourd psychologiquement. "

Comme nous pouvons le voir dans cet extrait d'entretien, l'engagement pour la crèche demande beaucoup de temps aux membres de ces commissions, il occupe leur agenda : « je travaillais à $80 \%$ à l'école et pourtant je n'avais plus de temps pour moi »(Kloé). Pour certains, il débordait même sur le travail professionnel, c'est le cas pour Anna, membre de la commission salariés, qui réalise des tâches pour la crèche à son bureau plusieurs heures par jour. Il occupe 58 - aussi, comme nous l'avons vu avec l'extrait d'entretien de Kloé, « la tête ». Pour Émilie, membre de la commission salariés, qui est par ailleurs, en tant que professionnelle, responsable d'une association culturelle où elle s'occupe de la gestion RH (Ressources humaines), l'engagement demandé est même devenu trop important : "Après deux années [alors que mon enfant pouvait faire une troisième année], j'ai quitté la crèche. Ça me faisait une double charge mentale, en plus de mon travail, et ça prenait beaucoup de temps. Il fallait faire trop de choses, avec un jeune enfant ça me semblait impossible. »

\section{Etre employeur « une responsabilité énorme » qui nécessite des compétences}

Comme nous l'avons vu, être employeur bénévole prend du temps et occupe l'esprit. Aussi, "c'est une responsabilité énorme, alors que tu manques de formation, que tu manques de temps pour faire les choses bien » (Anna, commission salariés). Comme l'explique Anna dans cet extrait d'entretien, la gestion de la structure est un véritable travail qui demande du temps, mais aussi des compétences. La bonne volonté ne suffit pas : certains parents l'ont constaté à leurs dépens, c'est le cas de Samy, qui en tant que co-président a participé au recrutement de RT 
et de RTA : « je me suis clairement planté au moment des recrutements, je n'ai aucune compétence là-dedans ».

Suite à des absences répétées et non justifiées de membres de l'équipe, Anna (com. salariés) et Kloé (co-présidente) interviennent lors d'une réunion d'équipe. Elles rappellent les difficultés qu'entrainent ces absences pour la continuité du service, que cela pèse sur les professionnelles présentes, mais aussi sur les parents qui doivent les remplacer au pied levé. Kloé se remémore : «Je ne me considérais pas comme employeur. La seule fois où je l'ai ressenti, je n'ai pas dormi. C'est une réunion où j'ai rappelé le cadre : si vous êtes en retard, vous appelez. Si vous êtes absente, il faut un justificatif. On avait énormément d'absence, j'ai rappelé que s'il n'y avait pas de justificatifs, il y aurait blâme et que ça pouvait finir par un licenciement. Car c'est les parents et l'équipe qui compensent les absences. J'avais appris le discours par cœur. J'ai répété les phrases que je devais dire pendant une journée entière, j'avais écrit tout mon texte. "

\section{Le rôle d'employeur bénévole nourrit par l'expérience professionnelle, et inversement}

Comme nous l'avons vu, les parents apprennent, s'entrainent dans le cas de Kloé, à occuper la fonction employeur. Pour la plupart, les parents investis au sein du bureau et de la commission salariés ne sont pas employeurs de métiers, même s'ils réinvestissent leurs connaissances professionnelles au sein de la crèche. C'est le cas de Camille, qui a été un pilier de la commission salariés et est fonctionnaire au ministère du Travail. Elle raconte avoir mobilisé son expérience professionnelle dans le cadre de la crèche : "J'ai mis en place plein de contrats aidés, car je connaissais par cœur ces dispositifs ". Émilie s'appuie aussi sur ses connaissances professionnelles, ainsi que sur son expérience personnelle, pour remplir les missions qui lui sont attribuées dans la commission salariés : « je connais bien le milieu associatif, les rapports entre salariés et bureau, le droit du travail, ce que c'est que gérer des salariés, d'avoir des responsabilités RH. [...] Mais aussi, je suis passée personnellement par un service civique, le RSA (Revenu de solidarité active), des contrats aidés, le chômage, l'intermittence, etc. Je suis passée par tous ces emplois qu'on avait à la crèche, j'ai vécu avec les mêmes salaires que les professionnelles qu'on embauchait. " 
Les parents ne sont cependant pas tous des professionnels de l'encadrement ou de la gestion du personnel : leurs connaissances de ces questions restent limitées. Mais pour Camille, les parents du bureau ou de la commission salariés " [sont] du milieu ", ils savent à qui s'adresser pour avoir des réponses à leurs questions. Comme elle l'explique : "On n'est pas formés, mais il y a plein de structures pour aider la fonction employeur dans les associations. On appelle notre organisme de formation, la plateforme d'appel du ministère du Travail, notre conseillère Pôle emploi. » Il n'est pas rare qu'ils s'appuient également sur les autres parents du collectif, où ils peuvent trouver des compétences spécifiques (connaissance du droit du travail, de la comptabilité, des relations publiques, de la petite enfance, etc.), ou encore " trouver du soutien [du côté] de l'ACEPRIF, d'anciens de Badaboum » (Samy, co-président).

Les parents investissent leurs compétences professionnelles et personnelles dans la crèche, mais l'inverse est aussi vrai. L'expérience associative est considérée par certains parents comme l'équivalent d'une véritable expérience de travail qui vient ainsi nourrir leurs curriculum vitae, où ils précisent cette expérience de "gestion $\mathrm{RH}$ ».

60 - C'est le cas notamment d'Anna, et de Camille qui explique avoir " gagné en confiance et compétences ". D'ailleurs, l'ACEPRIF ne s'y trompe pas et propose aux parents engagés dans des crèches parentales des formations pour "valoriser [ses] compétences de bénévoles. »

\section{La posture ambivalente des employeurs associatifs}

Être employeur bénévole, ce n'est pas seulement occuper un poste, donner du temps pour réaliser des tâches et utiliser ses compétences. C'est aussi avoir un rôle, occuper une posture spécifique et ambivalente.

\section{La difficile posture d'employeur, entre parents usagers et employeurs}

"Avoir la fonction RH, c'est avoir une relation particulière avec les salariés. Il faut être vigilant et rigoureux. Que toutes les personnes de la commission le soient aussi. Car les professionnelles s'occupent de ton enfant derrière. " (Camille, com. salariés) Les parents ayant en charge la fonction employeur pointent tous cette tension, la difficulté de leur posture : être à la fois usager ayant une forte proximité, des 
affinités, avec les salariées, du fait des présences hebdomadaires auprès des enfants qu'ils réalisent en commun. Et en même temps, être employeur, avec un rapport nécessairement distancié avec les salariées. Comme l'explique Samy, qui a été co-président de la crèche pendant un an, "cette double casquette n'est pas du tout protectrice. Tu es à la fois dans la position d'être en conflit en tant qu'usager, et en même temps tu peux aussi prendre des sanctions. [...] Le même jour, tu peux voir la RT comme président, comme employeur, et la rencontrer en tant que parent, car ton enfant a mordu un autre. "

Cette posture double amène parfois à réaliser des choix cornéliens, en particulier quand le parent employeur doit assumer des choix gestionnaires. C'est le cas de Samy : «ce que j'ai trouvé difficile, c'était lorsque la RT demandait de l'accompagnement psy. On avait envie de lui donner, mais on n'avait pas l'argent. Pareil quand on a voté des budgets où on faisait de gros efforts à la baisse, et que ça se répercutait sur les menus [des enfants et des professionnels]. C'était horrible. Mais il fallait assumer ces décisions. " (Samy, co-président)

Anna, de la commission salariés, fait une analyse similaire à celle de Samy : "Il y a deux casquettes. Sur le calcul des heures supplémentaires par exemple. En tant que parent, j'ai envie de dire aux professionnelles, de tout compter, mais en tant qu'employeur je trouve qu'elles abusent d'être à 5 minutes. " Elle ajoute : "Le fait que tu as tes enfants impactés, tu ne peux pas nier ou refuser la fonction employeur. Il faut que les meufs soient payées pour s'occuper de ton enfant. Point. La moindre erreur a des impacts sur ce que tu as de plus cher. Quand X enchaine les arrêts maladie et les retards, ça a des conséquences sur la fatigue des autres professionnelles, et ça a des conséquences sur le bienêtre de ton bébé."

Les entretiens soulignent tous l'ambiguïté de la posture de ceux qui occupent la fonction employeur, étant d'une part parents-usagersbénévoles proches des professionnelles d'autant plus qu'ils réalisent une activité en commun, et d'autre part employeurs devant faire respecter les engagements contractuels des salariées.

\section{Une responsabilité partagée}

Nous pouvons souligner une autre particularité de la posture employeur : celle-ci est partagée entre les individus qui la mettent en œuvre et le collectif. Les parents qui la mettent en œuvre n'ont pas de 
mandat pour la mettre en œuvre seuls, les décisions sont collectives. " J'avais beaucoup de responsabilités, mais sans pouvoir donner d'ordre. Je n'avais pas vraiment la main. Je n'étais pas cheffe. " (Anna, com. salariés) Camille, qui était dans la même commission, va dans le même sens : "c'est vraiment une expérience d'employeur, mais en même temps on n'avait que la délégation de la fonction. Car les décisions principales étaient validées par le collectif, en assemblée générale. C'était une fonction partagée. Ça a ses avantages et inconvénients, car ça dilue la décision, mais en même temps ça protège."

Dans les faits, toutes les décisions importantes ne sont pas prises par l'ensemble du collectif ou discutées en AG (par exemple un licenciement ou un recrutement). Elles sont néanmoins discutées collectivement à minima dans le cadre des commissions. Ainsi, la responsabilité est partagée. Ce n'est pas neutre sur l'exercice de la fonction employeur comme le raconte Émilie (com. salariés) : «Une de mes missions, c'était de suivre la responsable technique, notamment sur l'aspect bien-être au travail. Elle était en souffrance. La voir dans cet état c'était dur. [...] J'ai l'habitude de régler des problèmes de ce

62 - genre dans ma structure, mais là j'étais moins armée. C'est la petite enfance, ce n'est pas mon secteur professionnel. Je n'avais pas de point de comparaison avec d'autres crèches. Et après, si je vois des éléments intéressants à mettre en place, je n'ai aucun pouvoir pour le faire. Je dois convaincre le collectif. Et le collectif n'a pas eu les échanges que j'ai eus en tête à tête avec la RT, n'a pas la même relation de confiance avec elle. Il faut convaincre 15, 30 parents... et il y a peu d'espaces pour formuler, pour que le collectif s'approprie ces questions. Et puis, personne ne fait autorité, ne la légitime pour amener les gens à faire une expérience. [...] le bureau a beaucoup de responsabilités, mais il n'a pas beaucoup d'autorité. »

Ainsi, occuper la fonction employeur dans cette crèche parentale, c'est être chef sans être chef, avoir des responsabilités sans être responsable.

\section{Une légitimité limitée}

Nous pouvons repérer un dernier élément caractérisant la posture employeur. Les parents qui la mettent en œuvre disent manquer de légitimité, notamment pour discuter de l'organisation de la crèche et du travail face à une RT qui est, contrairement aux parents, une professionnelle du secteur de la petite enfance. C'est ce qu'explique 
Camille (com. salariés) : «La fonction RH ce n'est pas toujours évident quand tu travailles avec une RT qui a son mot à dire et avec qui tu n'es pas forcément d'accord. Elle c'est son métier, elle a la légitimité. Et parfois elle demandait des choses, comme le remplacement d'une salariée absente, dans l'intérêt des enfants, de ton enfant. Et toi tu dois avancer parfois des arguments un peu faibles. Tu ne peux pas recruter un salarié supplémentaire, car tu n'as pas les sous. "

Ce manque de légitimité des employeurs bénévoles face aux responsables salariées, est aussi alimenté par le turn-over des parents dans l'association. En effet, les parents restent en moyenne 2 à 3 ans dans la structure, et certaines salariées ont une ancienneté plus grande. Cela se traduit par le fait, qu'à chaque nouveau collectif de parents, à chaque renouvellement des commissions, les règles sont rediscutées. Comme l'explique Camille, " les règles sont toujours rebattues par la RT et la RTA. Alors on a travaillé sur la mise en place d'un règlement intérieur, pour sanctuariser les règles. "Conscients de cette difficulté, les parents de la crèche ont fixé les règles, définissant notamment une partie de l'organisation du travail dans la crèche, dans un règlement intérieur, afin d'éviter les éternelles négociations, mais permettant aussi d'assoir leur légitimité.

\section{Conclusion}

Cet article, réalisé à partir d'entretiens avec des parents d'une crèche associative et parentale, nous permet d'appréhender de manière compréhensive la manière dont ils occupent la fonction employeur, la manière dont ils vivent cette expérience. Nous avons vu qu'ils ne comprenaient véritablement ce qu'est être employeur qu'en le devenant, en exerçant la fonction employeur et en étant confrontés aux crises qui découlent de son exercice. Nous avons aussi remarqué que cette fonction employeur prend du temps et occupe l'esprit, nécessite des compétences (et que les parents les développent en travaillant dans leurs commissions). Nous avons également observé qu'occuper la fonction employeur c'est avoir une posture, tenir un rôle. Et que cela n'a rien d'évident : que cette posture est ambivalente. Ils sont responsables individuellement, mais cette responsabilité et les décisions sont partagées collectivement. Ils sont employeurs, mais leur légitimité d'employeurs de la petite enfance est limitée face aux 
professionnelles dont c'est le métier. Leur posture est également marquée par le fait qu'ils sont aussi à la fois usagers et employeurs. Ce dernier aspect est une particularité forte des crèches parentales : le fait qu'ils soient parents des enfants accueillis induit une dimension affective forte dans le rôle d'employeur qu'ils doivent endosser. Une singularité, qui crée des rapports hiérarchiques particuliers, et nécessite qu'ils trouvent une posture d'équilibre entre ces deux "casquettes ". Pour conclure, nous souhaiterions aborder un dernier point soulevé par les parents, mais que nous n'avons pas abordé précédemment. Lors des entretiens, ils pointent aussi le fait que la " crèche est sous financée. On n'a pas assez de sous pour salarier en Contrat à durée indéterminée (CDI). Il y a un manque de reconnaissance institutionnel, de ce que l'on fait. " (Émilie, com. salariés) De fait, ces bénévoles réalisent un travail, et le travail qu'ils réalisent est fortement dépendant des politiques publiques de la petite enfance et de l'emploi (Cottin-Marx, 2017; 2019). La forme des partenariats qu'ils réalisent avec les pouvoirs publics, les financements obtenus et leurs contraintes, ont des conséquences sur le travail de ces employeurs bénévoles. Ainsi, si regarder leur engagement comme un travail permet de voir les

64 - difficultés qu'ils ont à exercer le « métier » d'employeur bénévole, il serait intéressant d'enquêter sur les conséquences qu'entrainent les mutations de l'action publique sur " le métier de bénévole » (FerrandBechmann, 2000).

Simon Cottin-Marx est postdoctorant au LATTS, article réalisé avec le soutien de l'INJEP et de l'Institut français du monde associatif.

\section{Bibliographie}

Cottin-Marx, Simon, Professionnaliser pour « marchandiser » (et inversement), Thèse, Université Paris Est, 2016.

Cottin-Marx, Simon, « Les associations au service des politiques de l'emploi : genèse du dispositif local d'accompagnement ", Revue française d'administration publique, $\mathrm{n}^{\circ} 163,2017$, p. 557-570.

Cottin-Marx, Simon, Sociologie du monde associatif, Paris, La Découverte, 2019. 
Cottin-Marx, Simon, «Les relations de travail dans les entreprises associatives. Salariés et employeurs bénévoles face à l'ambivalence de leurs rôles ", La revue de l'IRES, n 101-102, 2020.

Ferrand-Bechmann, Dan, Le métier de bénévole, Paris, Anthropos, 2000. Hély, Matthieu, Les métamorphoses du monde associatif, Paris, PUF, 2009. Hély, Matthieu et Simonet, Maud, Le travail associatif, Paris, Presses universitaires de Paris Nanterre, 2013.

Hély, Matthieu; Rétif, Sophie et Simonet, Maud, «Figures de l'employeur et formes du «dialogue social » dans les entreprises de l'ESS », Mouvements, $\mathrm{n}^{\circ} 81,2015$, pp. 116-125.

Le Roy, Anne; Puissant, Emmanuelle; Devetter, François-Xaxier et Vatan, Sylvain, Économie politique des associations, Louvain la Neuve, DeBoeck, 2019.

Marchal, Emmanuelle, "L'entreprise associative entre calcul économique et désintéressement ", Revue française de sociologie, n 33-3, 1992, pp. 365-390. Offerlé, Michel, Patrons en France, Paris, La découverte, 2017.

Simonet, Maud, «Penser le bénévolat comme travail pour penser la sociologie du travail ", La revue de l'IRES, n 44, 2004, pp. 141-155.

Simonet, Maud, Le travail bénévole. Engagement citoyen ou travail gratuit?, Paris, La Dispute, 2010. 